\title{
Clinical Use and Utility of Amyloid Imaging
}

\author{
Henryk Barthel and Osama Sabri \\ Department of Nuclear Medicine, University of Leipzig, Leipzig, Germany
}

Currently, 3 amyloid PET tracers are approved and commercially available for clinical use. They allow for the accurate in vivo detection of amyloid plaques, one hallmark of Alzheimer disease. Here, we review the current knowledge on the clinical use and utility of amyloid imaging. Appropriate use criteria for the clinical application of amyloid imaging are established, and most currently available data point to their validity. Visual amyloid image analysis is highly standardized. Disclosure of amyloid imaging results is desired by many cognitively impaired subjects and seems to be safe once appropriate education is delivered to the disclosing clinicians. Regarding clinical utility, increasing evidence points to a change in diagnosis via amyloid imaging in about $30 \%$ of cases, to an increase in diagnostic confidence in about $60 \%$ of cases, to a change in patient management in about $60 \%$ of cases, and specifically to a change in medication in about $40 \%$ of cases. Also, amyloid imaging results seem to have a relevant impact on caregivers. Further, initial simulation studies point to a potential positive effect on patient outcome and to cost effectiveness of amyloid imaging. These features, however, will require confirmation in prospective clinical trials. More work is also required to determine the clinical utility of amyloid imaging specifically in subjects with mild cognitive impairment and in comparison with or in conjunction with other Alzheimer disease biomarkers. In summary, the clinical use of amyloid imaging is being studied, and the currently available data point to a relevant clinical utility of this imaging technique. Ongoing research will determine whether this accurate and noninvasive approach to amyloid plaque load detection will translate into a benefit to cognitively impaired subjects.

Key Words: amyloid; imaging; PET; dementia; utility

J Nucl Med 2017; 58:1711-1717

DOI: 10.2967/jnumed.116.185017

W

ith the successful development and subsequent clinical approval of ${ }^{18} \mathrm{~F}$-florbetapir, ${ }^{18} \mathrm{~F}$-florbetaben, and ${ }^{18} \mathrm{~F}$-flutemetamol, the nuclear imaging community has a set of $\beta$-amyloid aggregatetargeting PET tracers in hand for clinical use. They allow the in vivo detection or exclusion of neuritic $\beta$-amyloid plaques, one histopathologic hallmark in the neocortex of patients with Alzheimer disease (AD), which previously could be diagnosed only histopathologically after death. Of note, brain $\beta$-amyloid accumulation is known to be an early event in this disease (1) and is considered by many as the initial trigger of a cascade of other pathobiochemical and pathophysiologic

Received May 12, 2017; revision accepted Aug. 10, 2017.

For correspondence or reprints contact: Henryk Barthel, Department of Nuclear Medicine, University of Leipzig Hospital, Liebigstrasse 18, 04103 Leipzig, Germany.

E-mail: henryk.barthel@medizin.uni-leipzig.de

Published online Aug. 17, 2017.

COPYRIGHT (C) 2017 by the Society of Nuclear Medicine and Molecular Imaging. alterations finally leading to neurodegeneration and related cognitive decline in $\mathrm{AD}(2)$.

The emergence of amyloid imaging technology is hoped to fill a relevant diagnostic gap in the clinic in cognitively impaired subjects and in $\mathrm{AD}$ in particular. Most people would like to get a diagnosis when cognitive symptoms are identified (3). Conversely, dementia diagnosis is often missed and delayed $(4,5)$. With regard to the therapeutic implications of an AD diagnosis, it was reported that older people are willing to accept the relevant side effects of AD-modifying therapies (6). Also of interest, more than $80 \%$ of dementia specialists reported in 2013 that they would like to complement their portfolio of AD diagnosis tools by amyloid imaging (7).

The clinical approval of these 3 amyloid tracers was based on convincing results from phase 1-3 development programs. The phase 2 studies (comparison between patients with a clinical diagnosis and healthy controls) were designed rather similarly to the anticipated clinical-use scenarios of these tracers. However, the results of the phase 3 studies (comparison between in vivo PET imaging and postmortem histopathology) had limited applicability to routine clinical scenarios, mainly because of the end-of-life situation of the study cohorts. Thus, it is necessary to investigate the clinical utility of amyloid imaging in separate studies on clinical cohorts. On a further level and with imperative importance for reimbursement discussions, cost-efficacy analyses and data on the impact of amyloid imaging on clinical outcome are required.

Chiotis et al. (8) recently summarized the current knowledge on the clinical validity of amyloid PET imaging as an AD biomarker. For that purpose, they adapted a 5-phase oncology biomarker development framework. From their amyloid imaging literature review, they concluded that the aims of phases 1 (rationale for use) and 2 (discriminative ability) of the clinical validity testing have been achieved, and that the aim of phase 3 (early detection ability) has been achieved in part. Phase 4 research (performance in representative mild cognitive impairment subjects) was estimated to be in the ongoing state, and phase 5 research (quantification of impact and costs) was estimated to be still outstanding.

We have identified several recently published studies that have investigated the clinical use and utility of amyloid imaging. It is the aim of this article to provide an overview and a critical discussion on the current knowledge in this important field.

\section{CLINICAL USE OF AMYLOID IMAGING}

Amyloid imaging should in principle be able to provide an early $\mathrm{AD}$ diagnosis. On the basis of the amyloid cascade theory, the initial event in AD is amyloid buildup, which results in a cascade of other processes that ultimately lead to neurodegeneration and dementia (2). Furthermore, because many other dementia disorders, such as most forms of frontotemporal dementia, are amyloid-negative, amyloid imaging might contribute to the differential dementia diagnosis. 
This ability might even have therapeutic consequences, because it is known, for instance, that the $N$-methyl-D-aspartate receptor-blocking memantine has no systematic benefit in frontotemporal dementia (9). Of interest with regard to the amyloid cascade theory and the fact that there exist alternative theories of $\mathrm{AD}$ pathogenesis, such as the one claiming that tau aggregation is the cascade trigger, it was recently reported that the interpretation of amyloid images is not affected by belief about the pathogenic role of amyloid (10).

\section{Appropriate Use Criteria (AUCs) for Amyloid Imaging}

Bearing in mind the potential of amyloid imaging together with the ethical implications of potentially revealing a significant diagnosis without a cure, there were-quite early in the emergence of respective tracers-attempts to define scenarios in which amyloid imaging might be appropriate in future clinical use $(11,12)$. Vandenberghe et al. (12) stated that, to define appropriate use for amyloid imaging in a clinical setting, 3 factors need to be considered jointly: the clinical context, the health care system, and the societal perception of AD. Lately, with the regulatory approvals of the 3 amyloid tracers and the perspective of upcoming coverage decisions, it was felt that clear definitions must be made for the appropriate and inappropriate clinical use of amyloid imaging. As a consequence, AUCs were published in 2013 in a joint effort by the Society of Nuclear Medicine and Molecular Imaging and the Alzheimer's Association (13). These criteria stated that amyloid imaging is appropriate in subjects fulfilling all of the following 3 prerequisites: "Cognitive complaint with objectively confirmed impairment," "AD as a possible diagnosis, but when the diagnosis is uncertain after a comprehensive evaluation by a dementia expert," and "When knowledge of the presence or absence of $A \beta$ pathology is expected to increase diagnostic certainty and alter management." After a comprehensive literature review and expert discussion, these were filtered into 3 appropriate-use scenarios, and 6 inappropriate-use scenarios were also formulated (Supplemental Table 1; supplemental materials are available at http://jnm. snmjournals.org). These were soon afterward updated by a clarification of the dementia expert definition, as well as clarification of other aspects (14).

Of interest, Grundman et al. (15) investigated the clinical utility of amyloid imaging depending on whether the AUCs are met. They investigated 229 subjects imaged with ${ }^{18} \mathrm{~F}$-florbetapir. Although the impact of amyloid imaging on management plans was high in that study regardless of whether AUC-like or non-AUClike subjects were considered ( $88 \%$ vs. $86 \%$ ), a change in diagnosis via amyloid imaging was observed significantly more often in AUC-like than in non-AUC-like subjects (62\% vs. $45 \%)$. In contrast, Apostolova et al. (16) found that changes in diagnosis via amyloid imaging occurred less often in (AUC-consistent) earlyonset cases than in late-onset cases, and treatment changes occurred more often in the early-onset group.

\section{NOTEWORTHY}

- Clinical use of amyloid imaging is well defined and safe.

- Amyloid imaging changes diagnosis in about $30 \%$ of cases.

- Amyloid imaging increases diagnostic confidence in about $60 \%$ of cases.

- Amyloid imaging changes patient management in about $60 \%$ of cases.

- The impact of amyloid imaging on patient outcome is currently under investigation.
Nevertheless, the current AUCs seem to provide a reasonable framework for current clinical use of amyloid imaging. It is certainly necessary to further investigate this feature and to adapt the AUCs once new evidence on the usefulness or nonusefulness of amyloid imaging in certain scenarios emerges. For instance, Zwan et al. (17) reported that patients might benefit from amyloid imaging even if diagnostic confidence does not increase. Further, given a certain likelihood that disease-modifying antiamyloid drugs will be available in the future, AUCs might need to be expanded to cases in which clinical testing reveals typical AD features ("probable AD dementia"). That is to say, this patient cohort likewise comprises a relevant portion of amyloid-negative cases, which would not benefit from respective treatment (18).

\section{Amyloid Image Analysis}

The PET data of all 3 approved amyloid tracers are, in clinical routine, assessed visually. This is accomplished on a binary level; that is, the brains imaged are interpreted as either amyloid-positive or amyloid-negative. It is a requirement by the regulatory authorities that future amyloid PET readers first pass a tracer-specific training program. Because the amyloid tracers share similar uptake patterns, the respective training programs share some similarities: they all focus on teaching the identification of neocortical gray matter-versus-white matter contrast, with the loss of this contrast pointing to amyloid positivity and vice versa. The feasibility, accuracy, and reproducibility of the respective training programs were recently confirmed for all 3 tracers (19-21). One interesting question recently asked in this context relates to the potential incremental value of semiquantitative image analysis (22). In this regard, Pontecorvo et al. (21) showed that the consideration of SUV ratios during visual readings increases accuracy, especially in "below average" readers. More research on this topic is thus justified to fully understand the potential of combined visual and semiquantitative amyloid image analysis in a routine clinical scenario.

\section{Disclosure of Amyloid Imaging Results}

An interesting ethical discussion is ongoing regarding the disclosure of amyloid imaging results. This discussion aims at finding a much-desired balance between the "value of knowing" (23) and the principle of nonmaleficence ("avoiding potential harms of disclosure given the currently unproven clinical utility" (24)). Positive aspects of disclosing the results of amyloid imaging are the possibility of adapting the life plan and keeping autonomy, whereas negative aspects relate to fear of upsetting patients who have no therapeutic options. However, in support of disclosure, a general questionnaire study performed by Sullivan et al. (23) showed that, when asked about disclosure, $97 \%$ of more than 300 patients responded "I want to be told of serious, life-threatening diagnosis." More specifically, in another survey (25), 67\% of more than 2,600 adults in the United States and Europe responded that they would make use of a test for early AD diagnosis once such a test became available. In accordance, a metaanalysis of 23 studies in more than 9,000 subjects revealed that, in the case of the cognitive impairment subgroup, $84 \%$ of the participants favored disclosure of the dementia diagnosis to them (Fig. 1) (26). Regarding amyloid imaging, the majority of Alzheimer Disease Neuroimaging Initiative investigators who were surveyed in 2013 responded that they would, once respective tracers were approved by the Food and Drug Administration, disclose the results. This was paralleled by the notion that respective disclosure standards and training are required, together with respective outcome research (27). Other authors raised the concern that disclosing the results of amyloid imaging in binary categories as currently done might not provide the full 


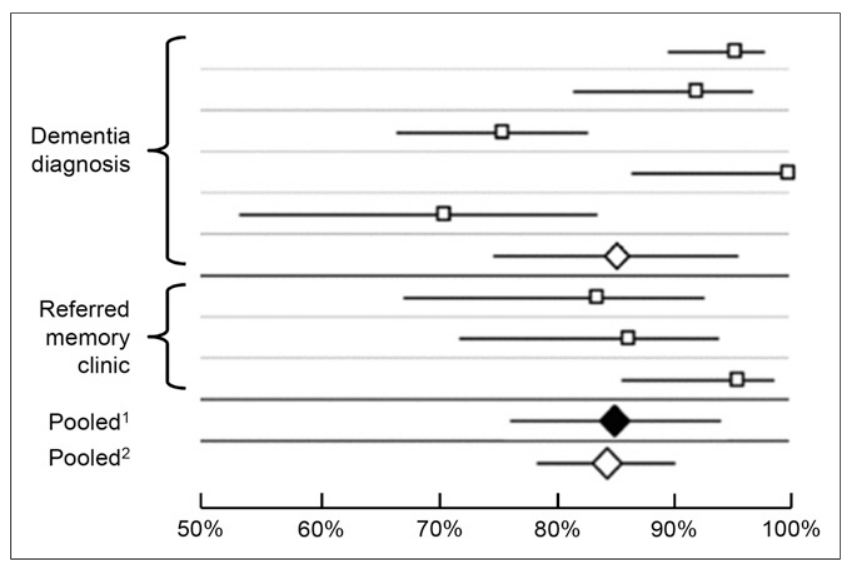

FIGURE 1. Results of metaanalysis investigating preference regarding disclosure of dementia diagnosis in subjects with cognitive impairment. ${ }^{1}$ Pooled percentage in favor, studies with response rate $\geq 75 \%$. ${ }^{2}$ Pooled percentage in favor, all studies. Figure is adapted from van den Dungen et al. (26).

picture (24), or pointed to the need for adequate education $(24,28,29)$. For cognitively impaired participants of research studies, Lingler et al. argued (30) that it is time to disclose the respective amyloid imaging results, again providing adequate pre- and postdisclosure education.

As a consequence, the same group recently developed and successfully tested educational materials for standardized pretest counseling and posttest disclosures in subjects with mild cognitive impairment (Supplemental Table 2) (31). Regardless, a recent investigation into the safety and tolerability of disclosing amyloid PET imaging results in 11 healthy older adults who took part in an AD biomarker trial revealed that disclosure did not significantly affect mood, subjective sense of memory impairment, perceived risk of developing $\mathrm{AD}$, or emotion. Also of note, amyloid-positive subjects were more likely to make positive lifestyle changes after disclosure than were amyloid-negative subjects (32). In good accordance, a similar study in 97 healthy older adults found no significant effect of disclosure on depressive symptoms or anxiety, despite a slight disclosure-related distress (33).

\section{CLINICAL UTILITY OF AMYLOID IMAGING}

The question of the clinical utility of amyloid imaging is of the utmost importance for the establishment of this new imaging technique in a routine clinical scenario. In principle, different levels of clinical utility exist for diagnostic tests, with different degrees of appreciation by patients/caregivers, medical doctors, and payers. These include change in diagnosis by the test, change in general diagnostic confidence by adding the test to the diagnostic regime, change in management, impact on caregivers, impact on patient outcome, and associated costs.

Sixteen studies concerning the clinical utility of amyloid PET imaging were found in the literature (17,34-48). Supplemental Table 3 summarizes these studies. Three studies address cost efficacy and will be discussed later in this article. Since 2012, altogether 1,360 subjects were investigated either prospectively or retrospectively, in either mono- or multicenter settings. While the earlier studies focused on ${ }^{11} \mathrm{C}$-Pittsburgh compound $\mathrm{B}$, in the following years the ${ }^{18} \mathrm{~F}$-labeled amyloid tracers were in the spotlight. Most studies included subjects with cognitive impairment (of either predementia or dementia degree) in whom a certain diagnostic uncertainty was evident after the standard diagnostic testing. Most cases included clinical testing and morphologic imaging, and some studies incorporated ${ }^{18} \mathrm{~F}-\mathrm{FDG}$ brain PET imaging, cerebrospinal fluid sampling for amyloid or tau, or APOe4 genotyping. PET positivity ranged from $39 \%$ to $90 \%$ in these studies. In 3 of these studies, the clinical value of amyloid imaging was studied together with that of ${ }^{18} \mathrm{~F}-\mathrm{FDG}$ brain PET imaging, and in 1 of these 3 studies this was further combined with repeated neuropsychologic testing (Supplemental Table 3).

\section{Impact of Amyloid Imaging on Diagnosis}

One hint of a clinical impact of amyloid imaging would be evidence of a change in diagnosis via imaging in a relevant portion of subjects investigated. Twelve studies with a total case number of 1,159 dealing with this question were found in the literature, the

TABLE 1

Clinical Utility of Amyloid PET Imaging

\begin{tabular}{lccccc}
\hline \multicolumn{1}{c}{ Study } & $\begin{array}{c}\text { Change in } \\
\text { diagnosis } \\
\text { (\% of cases) }\end{array}$ & $\begin{array}{c}\text { Gain in diagnostic } \\
\text { confidence } \\
\text { of cases) }\end{array}$ & $\begin{array}{c}\text { Gain in diagnostic } \\
\text { confidence } \\
\text { (on 0\%-100\% scale) }\end{array}$ & $\begin{array}{c}\text { Overall change } \\
\text { in management } \\
\text { (\% of cases) }\end{array}$ & $\begin{array}{c}\text { Change in } \\
\text { medication } \\
\text { (\% of cases) }\end{array}$ \\
\hline Frederiksen et al. 2012 (34) & 23 & 49 & - & - & - \\
\hline Schipke et al. 2012 (35) & - & 83 & - & 68 & - \\
\hline Degerman Gunnarsson et al. 2013 (37) & 30 & - & - & - & - \\
\hline Ossenkoppele et al. 2013 (38) & 23 & - & 16 & - & - \\
\hline Grundman et al. 2013 (39) & 55 & - & 22 & 87 & 31 \\
\hline Mitsis et al. 2014 (40) & 33 & - & - & - & - \\
\hline Sánchez-Juan et al. 2014 (41) & 9 & 8 & - & - & 35 \\
\hline Zannas et al. 2014 (42) & 72 & 36 & - & - & 45 \\
\hline Boccardi et al. 2016 (10) & 27 & - & 21 & - & 60 \\
\hline Bensaïdane et al. 2016 (45) & 32 & 44 & - & 71 & 39 \\
\hline Weston et al. 2016 (46) & 35 & 90 & - & 40 & 30 \\
\hline Schönecker et al. 2017 (47) & 21 & - & - & - & - \\
\hline Zwan et al. 2017 (17) & 19 & 87 & - & 37 & 24 \\
\hline Mean weighted by study cohort size & $\mathbf{2 9}$ & $\mathbf{6 3}$ & $\mathbf{2 0}$ & $\mathbf{6 4}$ & $\mathbf{3 8}$ \\
\hline
\end{tabular}


results of which are summarized in Table 1 . In all these studies, a relevant portion of subjects showed a diagnosis change via amyloid imaging, ranging from $9 \%$ to $72 \%$. The weighted (by the study cohort size) change in diagnosis was $29 \%$ of cases across all studies. For example, in a ${ }^{18} \mathrm{~F}$-florbetapir multicenter study on 229 cognitively impaired subjects with midrange, $15 \%-85 \%$, prescan diagnostic confidence of AD, Grundman et al. (39) found that amyloid imaging changed the diagnosis in all 3 prescan diagnosis groups ("due to AD," "indeterminate," "not due to AD") to a similar degree, obviously with different accentuation for the amyloid-positive subjects versus the amyloid-negative subjects (Fig. 2). Further information on interesting studies on this topic is provided in the supplemental material.

\section{Impact of Amyloid Imaging on Diagnostic Confidence}

The fact that amyloid imaging changes the diagnosis in a relevant number of cases does not, in the absence of a diagnostic gold standard in the routine clinical scenario, necessarily imply a significant benefit. Thus, it is of interest to also investigate how and to what degree amyloid imaging affects diagnostic confidence. Ten studies with a total of 1,297 cases were identified dealing with this matter, 7 of which reported the results on a case frequency basis and 3 on a $0 \%-100 \%$ scale basis. All studies reported a gain in diagnostic confidence via amyloid imaging. On a case frequency basis, the study results ranged from $8 \%$ to $90 \%$, whereas on a $0 \%-$ $100 \%$ scale basis the gain in diagnostic confidence was reported to be $16 \%, 22 \%$, and $21 \%$ (Table 1). Further information on interesting studies on this topic is provided in the supplemental material. Taking all relevant studies together, the weighted (by the study cohort size) gain in diagnostic confidence was $63 \%$ on a case frequency basis and $20 \%$ on a $0 \%-100 \%$ scale basis.

\section{Impact of Amyloid Imaging on Patient Management}

Although the impact on diagnosis establishment and diagnostic confidence by amyloid imaging is mainly of relevance to the patients and their caregivers ("value of knowing") and to the referring doctors, it is not regarded as sufficient evidence to justify reimbursement by many payers, who require evidence of a patient outcome effect. Of the different levels of evidence on the clinical utility of amyloid imaging, the impact on patient management assumes a medium degree. In the literature, we found 8 studies

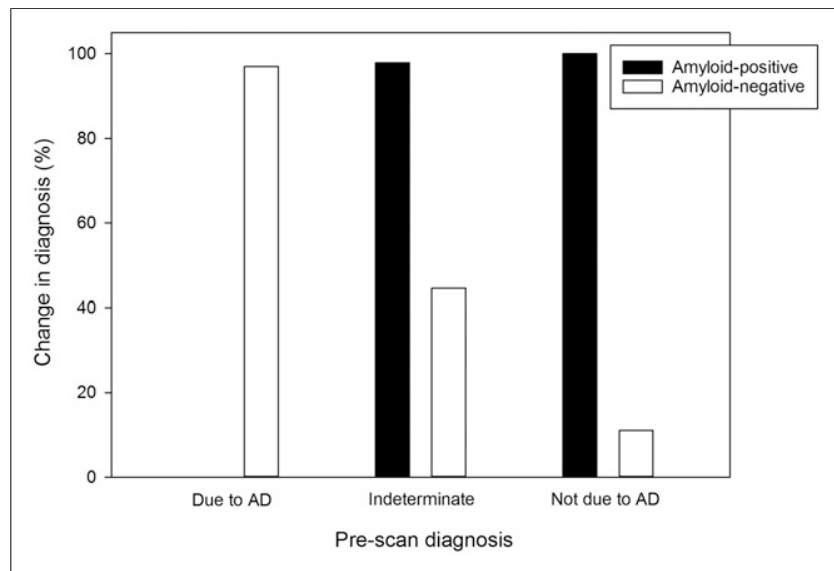

FIGURE 2. Change in diagnosis via amyloid imaging depending on prescan diagnosis and binary imaging outcome. Data to create this graph were taken from Grundman et al. (39). AD = Alzheimer disease. with a total case number of 1,068 investigating the effect of amyloid imaging on patient management. Five of these reported the effect on general management, and 7 of these more specifically reported the effect on medication management. The respective study results are summarized in Table 1 . All studies found amyloid imaging to have a relevant impact on management. The impact ranged between $37 \%$ and $87 \%$ of cases for overall change in management and between $24 \%$ and $60 \%$ of cases for change in medication. The weighted (by the study cohort size) change in overall management via amyloid imaging was $64 \%$ of cases, and the weighted change in medication, $38 \%$ of cases (Table 1). Further information on interesting studies on this topic is provided in the supplemental material.

\section{Impact of Amyloid Imaging on Caregivers}

Apart from the impact of amyloid imaging on the patients and their medical professionals, it is relevant to understand how this imaging directly (other than the indirect effects on the care plan) affects the patients' caregivers. Surprisingly, the direct impact of amyloid imaging on caregivers has not been systematically addressed so far. We could find only one publication addressing this question, by Bensaïdane et al. (45). In that single-center ${ }^{18} \mathrm{~F}$ NAV4694 study on 28 patients with atypical dementia (in the opinion of expert behavioral neurologists after reviewing history and basic lab, MRI, and ${ }^{18} \mathrm{~F}-\mathrm{FDG}$ PET results [PET positivity, $50 \%]$ ), their caregivers completed a questionnaire and were interviewed. All domains (anxiety, depression, disease perception, future anticipation, and quality of life) were positively affected - independent of the PET result—by disclosing the amyloid imaging finding, with a global impact of about $3.6 \pm 0.4$ on a $1-5$ Likert scale. Impressively, for the particular questions raised, "I appreciate every instant with my beloved one even more since we know the precise diagnosis" was most markedly acknowledged (45).

\section{Impact of Amyloid Imaging on Clinical Outcome}

The question of whether and to what extent amyloid imaging influences the clinical outcome of the subjects investigated is of great relevance, especially concerning reimbursement decisions. Providing respective positive evidence is, as with most other neurodegenerative disorders, complicated by the fact that there is still no cure available for AD. Instead, interventional efforts currently focus on drugs with potential symptomatic effects, changes in lifestyle, and reduction of cardiovascular risk factors.

To our knowledge, no clinical studies are yet available in the literature addressing whether amyloid imaging has a potential effect on clinical outcome. However, 3 model simulation studies on that topic have been published: Guo et al. (36) modeled the outcome effect of ${ }^{18} \mathrm{~F}$-florbetaben in predementia and dementia subjects. For both subgroups, that study found that amyloid imaging brought about estimated gains of 0.27 and 0.03 in quality-adjusted life years (QALYs; a measure combining the quality and the quantity of life lived), as compared with a scenario without amyloid imaging. Also, in the predementia group, amyloid imaging was associated with a better outcome regarding duration of the predementia phase, caregiver time, and other parameters. Hornberger et al. $(43,48)$ performed 2 similar simulations for ${ }^{18} \mathrm{~F}$-florbetapir PET in Spanish and French dementia cohorts. The QALY gains by amyloid imaging (likewise as compared with a scenario without amyloid imaging) estimated in these modeling studies were 0.008 and 0.021 , respectively. However, it will clearly be necessary for future studies to also investigate the simulation parameters in realworld clinical patients. 


\section{COST EFFECTIVENESS OF AMYLOID IMAGING}

From the health-economic point of view, it is important to investigate the costs by which the clinical utility of amyloid imaging is achieved. Relevant parameters in this context are the cost savings by the procedure, the costs per QALY, the cost-efficiency ratio (portion of subjects in whom amyloid imaging is cost-effective based on national willingness-to-pay thresholds for one QALY), and the incremental cost-effectiveness ratio (another parameter describing the relation between the costs and the effect of amyloid imaging). The 3 previously cited model simulation studies dealing with the effect of amyloid imaging on outcome also dealt with these matters: Guo et al. (36) simulated the cost effectiveness of ${ }^{18} \mathrm{~F}$ florbetaben in the United States, and Hornberger et al. $(43,48)$ performed a respective modeling for ${ }^{18} \mathrm{~F}$-florbetapir in Spain and France. In the study of Guo et al., cost savings by amyloid imaging were estimated at $\$ 13,018$ and $\$ 11,389$ per subject over a lifetime in predementia and dementia subjects, respectively. With a willingness-to-pay threshold of $\$ 50,000$ for one QALY, amyloid PET was cost-effective in $58 \%$ and $98 \%$ of the simulated predementia and dementia cases, respectively. In the 2 studies of Hornberger et al., it was estimated that amyloid imaging does not lead to any cost savings. However, the incremental cost-effectiveness ratios were $€ 4,769$ and $€ 21,888$, respectively. Further, with willingnessto-pay thresholds of $€ 30,000$ and $€ 40,000$, respectively, for one QALY, amyloid PET was cost effective in more than $82 \%$ and more than $95 \%$ of the simulated dementia cases, respectively. Like the clinical outcome data, the cost-effectiveness data will require future studies to investigate these simulated parameters on a clinical patient basis.

\section{FUTURE PERSPECTIVES}

The current literature on the clinical utility of amyloid imaging mainly focuses-in keeping with the AUCs-on clinically uncertain dementia cases, both of early onset and of late onset. More work is required to systematically determine the clinical benefit of amyloid imaging in mild cognitive impairment subjects also. This further work should consider the recently published Canadian consensus guidelines on the use of amyloid imaging, which stated that "As a general rule, amyloid PET could be considered in [mild cognitive impairment] patients from whom the dementia expert has determined that greater certainty about the underlying pathology would alter management." (49).

It will also be necessary to investigate the impact of special education for clinicians who handle amyloid imaging information, an important topic as recently pointed out by different groups (5052). This education should contain lines of arguments on why there is clinical utility to both PET imaging outcome scenarios (amyloid positivity and negativity).

For discussions with payers concerning reimbursement, more evidence from clinical outcome research is likewise required. The U.S. Centers for Medicare and Medicaid Services decided in 2013 to restrict amyloid imaging coverage by arguing that the evidence for clinical use was insufficient at that time. Although this decision was acknowledged by some authors (53), it was criticized by others as an example of a misbalance in the Medicare coverage criteria between drugs and diagnostics (54). However, instead of broadly covering amyloid imaging, Medicare decided to cover one amyloid scan per patient for those enrolled in a coverage-of-evidence program. This decision led to the rollout of the so-called IDEAS (Imaging Dementia-Evidence for Amyloid Scanning) study, which aims at providing systematic evidence of high quality for a positive outcome effect of amyloid imaging. In that open-label, longitudinal cohort study, subjects fulfilling the AUCs will be imaged. Two primary objectives will be tested, namely the change in management via amyloid PET imaging and the impact of amyloid-state knowledge via PET imaging on hospital admissions and emergency room visits. As of May 4, 2017, 911 sites were active in that study, and 9,615 PET scans had been performed. The aim is to complete the study data analysis by 2020 , with the hope that, if the outcome of the study is positive, the Centers for Medicare and Medicaid Services will revise its coverage decision by 2022 (55). As a European multicenter initiative with similar aims, the so-called AMYPAD (Amyloid Imaging to Prevent Alzheimer's Disease) study will soon also address the clinical relevance of amyloid imaging. Among several different study objectives, AMYPAD will-in mild cognitive impairment subjects and dementia patients-determine the impact of amyloid imaging on change in diagnosis depending on whether PET is used early or late in the diagnostic process. Interestingly, this study will be paralleled by a change-in-management investigation in so-called SCD-plus (subjective cognitive decline with additional evidence for preclinical AD) subjects (56). All these efforts will provide the missing evidence on the clinical validity of amyloid imaging (8).

Finally, it would be interesting to combine research on the clinical utility of amyloid PET imaging with that on MRI. This research is relevant because brain MRI often represents first-line imaging in cognitive impairment and because hybrid amyloid PET/ MRI might be used more often—when available — instead of MRI alone in the future $(57,58)$. Also, as soon as tau PET imaging becomes more broadly available, research should investigate the gain in clinical value by adding this technique to amyloid imaging. Last but not least, and with major relevance regarding cost-efficacy discussions, the clinical utility of amyloid imaging should be systematically compared with that of amyloid cerebrospinal fluid measurements.

\section{SUMMARY AND CONCLUSION}

AUCs for the clinical application of amyloid imaging have been established, and the currently available literature points to their validity. Also, visual amyloid image analysis is highly standardized. Disclosure of amyloid imaging results is desired by many cognitively impaired subjects and seems to be safe once appropriate education is delivered to the disclosing clinicians. Regarding clinical utility, increasing evidence points to a change in diagnosis via amyloid imaging in about $30 \%$ of cases, to an increase in diagnostic confidence in about $60 \%$ of cases, to a change in patient management in about $60 \%$ of cases, and specifically to a change in medication in about $40 \%$ of cases. Also, the results of amyloid imaging seem to have a relevant impact on caregivers. Further, initial simulation studies point to a potential positive effect on patient outcome and the cost effectiveness of amyloid imaging. These features, however, will require confirmation in prospective clinical trials. More work is also required to determine the clinical utility of amyloid imaging specifically in subjects with mild cognitive impairment and in comparison with or in conjunction with other AD biomarkers.

The clinical use of amyloid imaging is subject to ongoing investigations, and the currently available data point to a relevant clinical utility for this imaging technique. Ongoing research will 
tell whether this utility will translate into a benefit to patients. By that means, this research will answer the question of whether we will be able to fully tap the potential of this accurate approach to noninvasively determining amyloid plaque load in the clinical field.

\section{DISCLOSURE}

Henryk Barthel and Osama Sabri received research support, consultant honoraria, and travel expenses from Piramal Imaging, Berlin, Germany. No other potential conflict of interest relevant to this article was reported.

\section{ACKNOWLEDGMENT}

We thank Damian McLeod for proofreading the manuscript.

\section{REFERENCES}

1. Braak H, Braak E. Neuropathological stageing of Alzheimer-related changes. Acta Neuropathol (Berl). 1991;82:239-259.

2. Jack CR Jr, Knopman DS, Jagust WJ, et al. Hypothetical model of dynamic biomarkers of the Alzheimer's pathological cascade. Lancet Neurol. 2010; 9:119-128.

3. Public attitudes about diagnosis: value of knowing. Alzheimer Europe website. http://www.alzheimer-europe.org/Research/Value-of-Knowing/Publicattitudes-about-diagnosis. Updated July 13, 2011. Accessed August 21, 2017.

4. Bradford A, Kunik ME, Schulz P, Williams SP, Singh H. Missed and delayed diagnosis of dementia in primary care: prevalence and contributing factors. Alzheimer Dis Assoc Disord. 2009;23:306-314.

5. Bradford A, Upchurch C, Bass D, et al. Knowledge of documented dementia diagnosis and treatment in veterans and their caregivers. Am J Alzheimers Dis Other Demen. 2011;26:127-133.

6. Hauber AB, Johnson FR, Fillit H, et al. Older Americans' risk-benefit preferences for modifying the course of Alzheimer disease. Alzheimer Dis Assoc Disord. 2009;23:23-32.

7. Klein EP, Kaye J. Dementia specialists and early adoption of amyloid imaging. J Alzheimers Dis. 2013;33:445-450.

8. Chiotis K, Saint-Aubert L, Boccardi M, et al. Clinical validity of increased cortical uptake of amyloid ligands on PET as a biomarker for Alzheimer's disease in the context of a structured 5-phase development framework. Neurobiol Aging. 2017;52:214-227.

9. Boxer AL, Knopman DS, Kaufer DI, et al. Memantine in patients with frontotemporal lobar degeneration: a multicentre, randomised, double-blind, placebocontrolled trial. Lancet Neurol. 2013;12:149-156.

10. Boccardi M, Altomare D, Ferrari C, et al. Do beliefs about the pathogenetic role of amyloid affect the interpretation of amyloid PET in the clinic? Neurodegener Dis. 2016;16:111-117.

11. Laforce R Jr, Rabinovici GD. Amyloid imaging in the differential diagnosis of dementia: review and potential clinical applications. Alzheimers Res Ther. 2011;3:31

12. Vandenberghe R, Adamczuk K, Dupont P, Laere KV, Chételat G. Amyloid PET in clinical practice: its place in the multidimensional space of Alzheimer's disease. Neuroimage Clin. 2013;2:497-511.

13. Johnson KA, Minoshima S, Bohnen NI, et al. Appropriate use criteria for amyloid PET: a report of the Amyloid Imaging Task Force, the Society of Nuclear Medicine and Molecular Imaging, and the Alzheimer's Association. J Nucl Med. 2013;54:476-490

14. Johnson KA, Minoshima S, Bohnen NI, et al. Update on appropriate use criteria for amyloid PET imaging: dementia experts, mild cognitive impairment, and education. J Nucl Med. 2013;54:1011-1013.

15. Grundman M, Johnson KA, Lu M, et al. Effect of amyloid imaging on the diagnosis and management of patients with cognitive decline: impact of appropriate use criteria. Dement Geriatr Cogn Disord. 2016;41:80-92.

16. Apostolova LG, Haider JM, Goukasian N, et al. Critical review of the appropriate use criteria for amyloid imaging: effect on diagnosis and patient care. Alzheimers Dement (Amst). 2016;5:15-22.

17. Zwan MD, Bouwman FH, Konijnenberg E, et al. Diagnostic impact of $\left.{ }^{18} \mathrm{~F}\right]$ flutemetamol PET in early-onset dementia. Alzheimers Res Ther. 2017;9:2.
18. Barthel H, Seibyl J, Sabri O. The role of positron emission tomography imaging in understanding Alzheimer's disease. Expert Rev Neurother. 2015;15:395-406.

19. Buckley CJ, Sherwin PF, Smith AP, Wolber J, Weick SM, Brooks DJ. Validation of an electronic image reader training programme for interpretation of $\left[{ }^{18} \mathrm{~F}\right]$ flutemetamol $\beta$-amyloid PET brain images. Nucl Med Commun. 2017;38:234241.

20. Seibyl J, Catafau AM, Barthel H, et al. Impact of training method on the robustness of the visual assessment of ${ }^{18} \mathrm{~F}$-florbetaben PET scans: results from a phase3 study. J Nucl Med. 2016;57:900-906.

21. Pontecorvo MJ, Arora AK, Devine M, et al. Quantitation of PET signal as an adjunct to visual interpretation of florbetapir imaging. Eur J Nucl Med Mol Imaging. 2017;44:825-837.

22. Barthel H, Seibyl J, Sabri O. Yes we can analyse amyloid images: now what? Eur J Nucl Med Mol Imaging. 2017;44:822-824.

23. Sullivan K, O'Conor F. Should a diagnosis of Alzheimer's disease be disclosed? Aging Ment Health. 2001;5:340-348.

24. Roberts JS, Dunn LB, Rabinovici GD. Amyloid imaging, risk disclosure and Alzheimer's disease: ethical and practical issues. Neurodegener Dis Manag. 2013;3:219-229.

25. Wikler EM, Blendon RJ, Benson JM. Would you want to know? Public attitudes on early diagnostic testing for Alzheimer's disease. Alzheimers Res Ther. 2013;5:43.

26. van den Dungen P, van Kuijk L, van Marwijk H, et al. Preferences regarding disclosure of a diagnosis of dementia: a systematic review. Int Psychogeriatr. 2014;26:1603-1618

27. Shulman MB, Harkins K, Green RC, Karlawish J. Using AD biomarker research results for clinical care: a survey of ADNI investigators. Neurology. 2013;81:11141121.

28. Leuzy A, Zimmer ER, Heurling K, Rosa-Neto P, Gauthier S. Use of amyloid PET across the spectrum of Alzheimer's disease: clinical utility and associated ethical issues. Amyloid. 2014;21:143-148.

29. Grill JD, Apostolova LG, Bullain S, et al. Communicating mild cognitive impairment diagnoses with and without amyloid imaging. Alzheimers Res Ther. 2017;9:35.

30. Lingler JH, Klunk WE. Disclosure of amyloid imaging results to research participants: has the time come? Alzheimers Dement. 2013;9:741.e2-744.e2.

31. Lingler JH, Butters MA, Gentry AL, et al. Development of a standardized approach to disclosing amyloid imaging research results in mild cognitive impairment. J Alzheimers Dis. 2016;52:17-24.

32. Lim YY, Maruff P, Getter C, Snyder PJ. Disclosure of positron emission tomography amyloid imaging results: a preliminary study of safety and tolerability. Alzheimers Dement. 2016;12:454-458.

33. Burns JM, Johnson DK, Liebmann EP, Bothwell RJ, Morris JK, Vidoni ED. Safety of disclosing amyloid status in cognitively normal older adults. Alzheimers Dement. March 3, 2017 [Epub ahead of print].

34. Frederiksen KS, Hasselbalch SG, Hejl AM, Law I, Højgaard L, Waldemar G. Added diagnostic value of ${ }^{11} \mathrm{C}$-PiB-PET in memory clinic patients with uncertain diagnosis. Dement Geriatr Cogn Dis Extra. 2012;2:610-621.

35. Schipke CG, Peters O, Heuser I, et al. Impact of beta-amyloid-specific florbetaben PET imaging on confidence in early diagnosis of Alzheimer's disease. Dement Geriatr Cogn Disord. 2012;33:416-422.

36. Guo S, Getsios D, Hernandez L, et al. Florbetaben PET in the early diagnosis of Alzheimer's disease: a discrete event simulation to explore its potential value and key data gaps. Int J Alzheimers Dis. 2012:548157.

37. Degerman Gunnarsson M, Lindau M, Santillo AF, et al. Re-evaluation of clinical dementia diagnoses with Pittsburgh compound B positron emission tomography. Dement Geriatr Cogn Dis Extra. 2013;3:472-481.

38. Ossenkoppele R, Prins ND, Pijnenburg YA, et al. Impact of molecular imaging on the diagnostic process in a memory clinic. Alzheimers Dement. 2013;9:414421.

39. Grundman M, Pontecorvo MJ, Salloway SP, et al. Potential impact of amyloid imaging on diagnosis and intended management in patients with progressive cognitive decline. Alzheimer Dis Assoc Disord. 2013;27:4-15.

40. Mitsis EM, Bender HA, Kostakoglu L, et al. A consecutive case series experience with $\left[{ }^{18} \mathrm{~F}\right]$ florbetapir PET imaging in an urban dementia center: impact on quality of life, decision making, and disposition. Mol Neurodegener. 2014;9:10.

41. Sánchez-Juan P, Ghosh PM, Hagen J, et al. Practical utility of amyloid and FDGPET in an academic dementia center. Neurology. 2014;82:230-238.

42. Zannas AS, Doraiswamy PM, Shpanskaya KS, et al. Impact of ${ }^{18} \mathrm{~F}$-florbetapir PET imaging of $\beta$-amyloid neuritic plaque density on clinical decision-making. Neurocase. 2014;20:466-473.

43. Hornberger J, Michalopoulos S, Dai M, Andrade P, Dilla T, Happich M. Costeffectiveness of florbetapir-PET in Alzheimer's disease: a Spanish societal perspective. J Ment Health Policy Econ. 2015;18:63-73. 
44. Boccardi M, Altomare D, Ferrari C, et al. Assessment of the incremental diagnostic value of florbetapir F18 imaging in patients with cognitive impairment: the Incremental Diagnostic Value of Amyloid PET with $\left[{ }^{18} \mathrm{~F}\right]$-Florbetapir (INDIA-FBP) Study. JAMA Neurol. 2016;73:1417-1424.

45. Bensaïdane MR, Beauregard JM, Poulin S, et al. Clinical utility of amyloid PET imaging in the differential diagnosis of atypical dementias and its impact on caregivers. J Alzheimers Dis. 2016;52:1251-1262.

46. Weston PS, Paterson RW, Dickson J, et al. Diagnosing dementia in the clinical setting: can amyloid PET provide additional value over cerebrospinal fluid? J Alzheimers Dis. 2016;54:1297-1302.

47. Schönecker S, Prix C, Raiser T, et al. Amyloid positron-emission-tomography with $\left[{ }^{18} \mathrm{~F}\right]$-florbetaben in the diagnostic workup of dementia patients. Nervenarzt. 2017;88:156-161.

48. Hornberger J, Bae J, Watson I, Johnston J, Happich M. Clinical and cost implications of amyloid beta detection with amyloid beta positron emission tomography imaging in early Alzheimer's disease: the case of florbetapir. Curr Med Res Opin. 2017;33:675-685.

49. Laforce R, Rosa-Neto P, Soucy JP, Rabinovici GD, Dubois B, Gauthier S. Canadian consensus guidelines on use of amyloid imaging in Canada: update and future directions from the specialized task force on amyloid imaging in Canada. Can J Neurol Sci. 2016;43:503-512.
50. Zhong Y, Karlawish J, Johnson MK, Neumann PJ, Cohen JT. The potential value of $\beta$-amyloid imaging for the diagnosis and management of dementia: a survey of clinicians. Alzheimer Dis Assoc Disord. 2017;31:27-33.

51. Ganz ML, Tawah AF, Guo S, et al. The impact of $\beta$-amyloid positron emission tomography on the diagnostic and treatment decisions of dementia experts. Neurodegener Dis Manag. 2017;7:107-117.

52. Witte MM, Foster NL, Fleisher AS, et al. Clinical use of amyloid-positron emission tomography neuroimaging: practical and bioethical considerations. Alzheimers Dement (Amst). 2015;1:358-367.

53. Steinbrook R. The Centers for Medicare \& Medicaid Services and amyloid- $\beta$ positron emission tomography for Alzheimer disease. JAMA Intern Med. 2014;174:135.

54. Cohen JP, Dong J, Lu CY, Chakravarthy R. Restricting access to florbetapir: Medicare coverage criteria for diagnostics and drugs are inconsistent. BMJ. 2015;351:h3333.

55. Home page. iDEAS website. www.ideas-study.org. Accessed August 21, 2017

56. Home page. AMYPAD website. www.amypad.eu. Accessed August 21, 2017.

57. Teipel S, Drzezga A, Grothe MJ, et al. Multimodal imaging in Alzheimer's disease: validity and usefulness for early detection. Lancet Neurol. 2015;14:1037-1053.

58. Schütz L, Lobsien D, Fritzsch D, et al. Feasibility and acceptance of simultaneous amyloid PET/MRI. Eur J Nucl Med Mol Imaging. 2016;43:2236-2243. 